\title{
Subtração de imagens para detecção de mudanças na cobertura vegetal da bacia hidrográfica do Rio Alegre - Alegre/ES
}

\author{
Kennedy Ribeiro da Silva ${ }^{1}$ \\ Daiani Bernardo Pirovani ${ }^{1}$ \\ Larice Nogueira de Andrade ${ }^{2}$ \\ Alexandre Rosa dos Santos ${ }^{1}$ \\ Roberto Avelino Cecílio ${ }^{1}$ \\ João Paulo Bestete ${ }^{1}$
}

\begin{abstract}
${ }^{1}$ Universidade Federal do Espírito Santo - UFES/ Departamento de Engenharia Florestal, Programa de Pós-Graduação em Ciências Florestais, Avenida Carlos Lindemberg, s/n, Centro, Jerônimo Monteiro - ES, Brasil.

${ }^{2}$ Universidade Federal do Espírito Santo - UFES/ Departamento de Engenharia Ambiental, Programa de Pós-Graduação em Engenharia Ambiental, Centro Tecnológico, UFES, Vitória ES, Brasil.
\end{abstract}

kennedyfloresta03@hotmail.com; daianipirovani@hotmail.com; lariceandrade@gmail.com; joaopaulobestete@bol.com.br; mundogeomatica@yahoo.com.br.

\begin{abstract}
This paper applies the index of high resolution vegetation (NDVI) in the vegetation change detection for the Alegre river hydrografic basin (Alegre - ES). Analyses were made by using CBERS sensors images from August 2004 to July 2008. The obtained results detection little land-cover changes, suggesting a preserved vegetation cover.
\end{abstract}

Palavras-chave: NDVI, sensoriamento remoto, change detection, CBERS.

\section{Introdução}

O mapeamento e monitoramento da cobertura vegetal receberam considerável impulso nas últimas décadas, com o advento do sensoriamento remoto, e processamento digital de imagens. $\mathrm{O}$ avanço nas pesquisas, a geração de novos sensores orbitais, e sua distribuição de forma mais acessível aos usuários, torna as imagens de satélite um dos produtos do sensoriamento remoto mais utilizado para análise da cobertura terrestre. Essas tecnologias contribuem para o mapeamento, monitoramento, fiscalização e controle da cobertura vegetal de extensas áreas da superfície terrestre (Ponzoni 2001).

Segundo Florenzano (2002), as imagens de satélite proporcionam uma visão sinóptica (de conjunto) e multitemporal (de dinâmica) de extensas áreas da superfície terrestre. Moreira (2005) afirma que os dados de reflectância dos alvos podem ser transformados em índices de vegetação, que foram criados com o intuito de ressaltar o comportamento espectral da vegetação em relação ao solo e a outros alvos da superfície terrestre, sendo o NDVI (Normalized Difference Vegetation Index) um dos índices mais utilizados.

A utilização de imagens de sensores remotos e a aplicação destes índices, em particular, o NDVI, são importantes na análise da dinâmica temporal da vegetação, geralmente associada ao crescimento das plantas. Relação empírica entre o ciclo climático anual e a variação sazonal do NDVI foi analisada em nível global por Potter e Brooks (1998). Estudos deste tipo existem em regiões semi-áridas da África (Justice et al., 1991; Nicholson e Farrar, 1994) e em ecossistemas brasileiros de cerrado (Assad et al., 1996) e de floresta amazônica (Almeida e Batista, 1998). 
Diante do exposto e da potencialidade das imagens multi-temporais na captura da dinâmica da paisagem, este trabalho tem por objetivo analisar as mudanças na cobertura vegetal da bacia hidrográfica do Rio Alegre.

\section{Metodologia}

A área de estudo compreende a bacia hidrográfica do Rio Alegre situada entre os

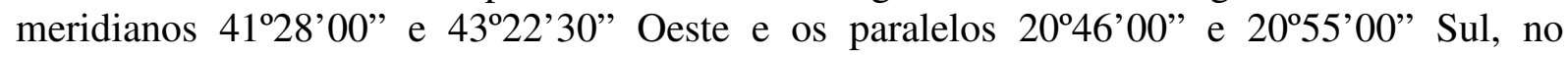
município de Alegre, no Estado do Espírito Santo (Figura 1). O Clima da região de estudo, de acordo com a classificação climática de Köppen, está enquadrado no tipo Cwa. Trata-se de um clima tropical de altitude, marcado por inverno seco e verão chuvoso.

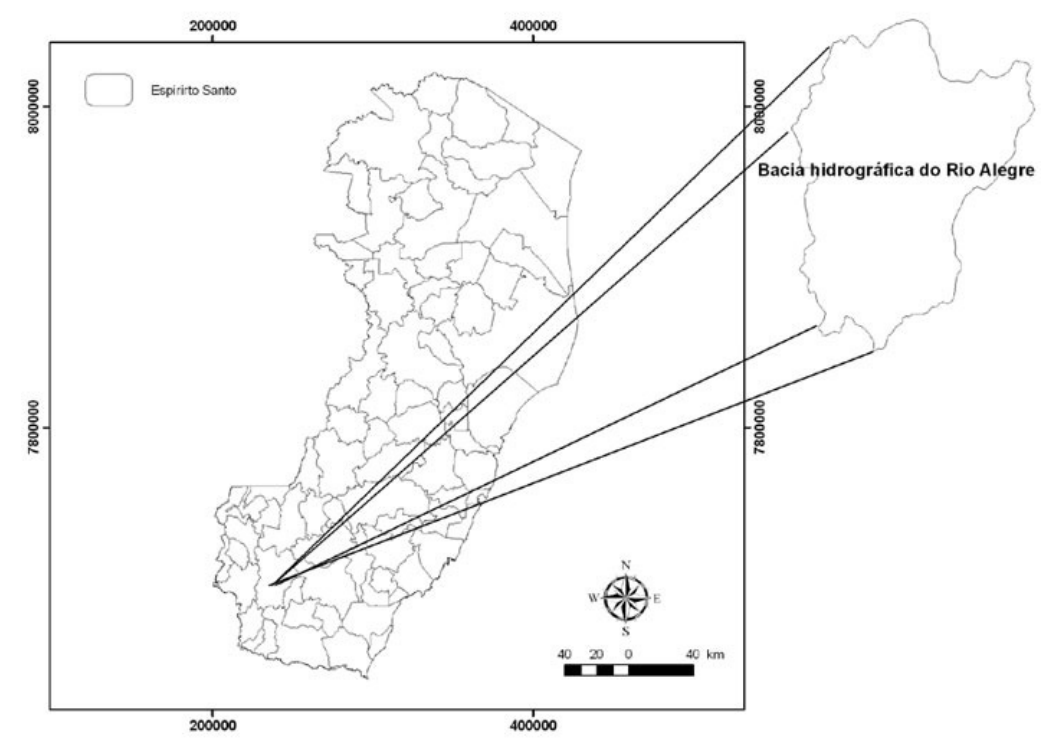

Figura 1. Área de estudo.

A análise das mudanças na cobertura vegetal foi realizada entre o período de agosto de 2004 a julho de 2008. O material utilizado foi composto pelos seguintes itens:

- Imagens Orbitais CCD/CBERS 2, de 11/08/2004 e 21/07/2008, órbita-ponto, 149-123;

- Sistema de Processamento de Informações Georreferenciadas - SPRING v 5.0 (DPI/INPE);

- Software de sistema de informações geográficas ArcGis 9.2/arcinfo;

O índice de vegetação (NDVI) utilizado neste trabalho (Equação 1) apresenta como variáveis as bandas do vermelho (banda 3 ) e do infravermelho próximo (banda 4).

$$
N D V I: \frac{I V P-V}{I V P+V}
$$

Onde,

IVP : valor da reflectância da banda no Infravermelho próximo

$V$ : valor de reflectância da banda no vermelho

Para compatibilizar os níveis de cinza dos elementos de imagem em cada banda espectral envolvidas no processamento foi utilizado o método da Uniformização das Médias e Variâncias - UMV. Este método consiste em igualar as médias e as variâncias de duas 
imagens através de uma transformação linear. Para isso, são calculados os parâmetros (ganho e offset) da função preservando, na imagem resultante, a média e a variância da imagem de referência. O cálculo do ganho e offset são baseados na função de transformação $f(x)=a x+b$, ou seja:

$$
\mu_{R}=\text { ganho } * \mu_{S}+\text { offset e } \sigma_{R}^{2}=\text { ganho }^{2} * \sigma_{s}^{2}
$$

Na uniformização das médias e variâncias, a imagem de 21/07/2008 foi utilizada como referência $(R)$, e a de 11/08/2004 como imagem de ajuste $(S)$. Em seguida, foram calculados os valores de ganho (Equação 2) e offset (Equação 3) aplicados às bandas 3 e 4 da imagem de ajuste $(S)$ (Equação 4).

$$
\begin{gathered}
\text { Ganho }=\sqrt{\sigma_{R}^{2} / \sigma_{S}^{2}} \\
\text { Offset }=\mu_{R}-\left(\sqrt{\sigma_{R}^{2} / \sigma_{S}^{2}} * \mu_{S}\right)
\end{gathered}
$$

Onde:

$\sigma_{R}^{2}$ : Variância dos níveis de cinza da imagem 21/07/2008.

$\sigma_{S}^{2}$ : Variância dos níveis de cinza da imagem 11/08/2004.

$\mu_{R}:$ Média dos níveis de cinza da imagem 21/07/2008.

$\mu_{s}:$ Média dos níveis de cinza da imagem 11/08/2004.

$$
S^{\prime}=S * \text { ganho }+ \text { offset }
$$

Com o intuito de realçar a vegetação dos demais alvos da área, reduzindo ao mínimo a influência do solo, foram geradas as imagens NDVI de 11/08/2004 e de 21/07/2008, a partir da reflectância correspondente às bandas 3 e 4, utilizando a Equação 1. Para avaliar as mudanças ocorridas entre o período estudado, foi realizada a subtração de imagens de acordo com a Equação 5.

$$
C=(A-B)
$$

Onde:

$C=$ Imagem-subtração

$A=$ Imagem NDVI correspondente à data 21/07/2008

$B=$ Imagem NDVI correspondente à data 11/08/2004

A utilização dos valores de ganho e offset (Equação 5) é importante para deslocar os níveis de cinza da imagem para valores positivos, evitando assim a compressão dos dados e a perda de informação. O resultado dessa subtração foi à imagem NDVI-04-08, na qual foram retiradas as estatísticas dos níveis de cinza de seu histograma: média $\mu=77,56$; variância $\sigma^{2}=25,80$ e desvio padrão $\sigma=5,08$. Esses valores foram necessários para a definição das classes temáticas apresentadas na Tabela 1 . 
Tabela 1- Limites das classes temáticas

\begin{tabular}{ll}
\hline \multicolumn{1}{c}{ Classes } & \multicolumn{1}{c}{ Níveis de Cinza } \\
\hline Desmatamento & 0 a $\mu-\sigma(0$ a 72,48) \\
Não mudança & $\mu-\sigma$ a $\mu+\sigma(72,48$ a 82,63$)$ \\
Regeneração & $\mu+\sigma$ a $255(82,63$ a 255$)$ \\
\hline
\end{tabular}

\section{Resultados Obtidos}

Na Tabela 2 são apresentados os valores de ganho $e$ offset obtidos a partir das Equações 2 e 3. As imagens ajustadas de acordo com a Equação 4, submetidas ao método de uniformização de médias e variâncias, compatibilizando as estatísticas dos níveis de cinza com a imagem de referência, são apresentadas na Figura 2.

Tabela 2 - Valores de ganho e offset para as bandas 3 e 4 da imagem CBERS-CCD.

\begin{tabular}{lcc}
\hline & Ganho & Offset \\
\hline Banda 3-2004 & 0,430 & 12,261 \\
Banda 4-2004 & 0,939 & $-24,97$ \\
\hline
\end{tabular}
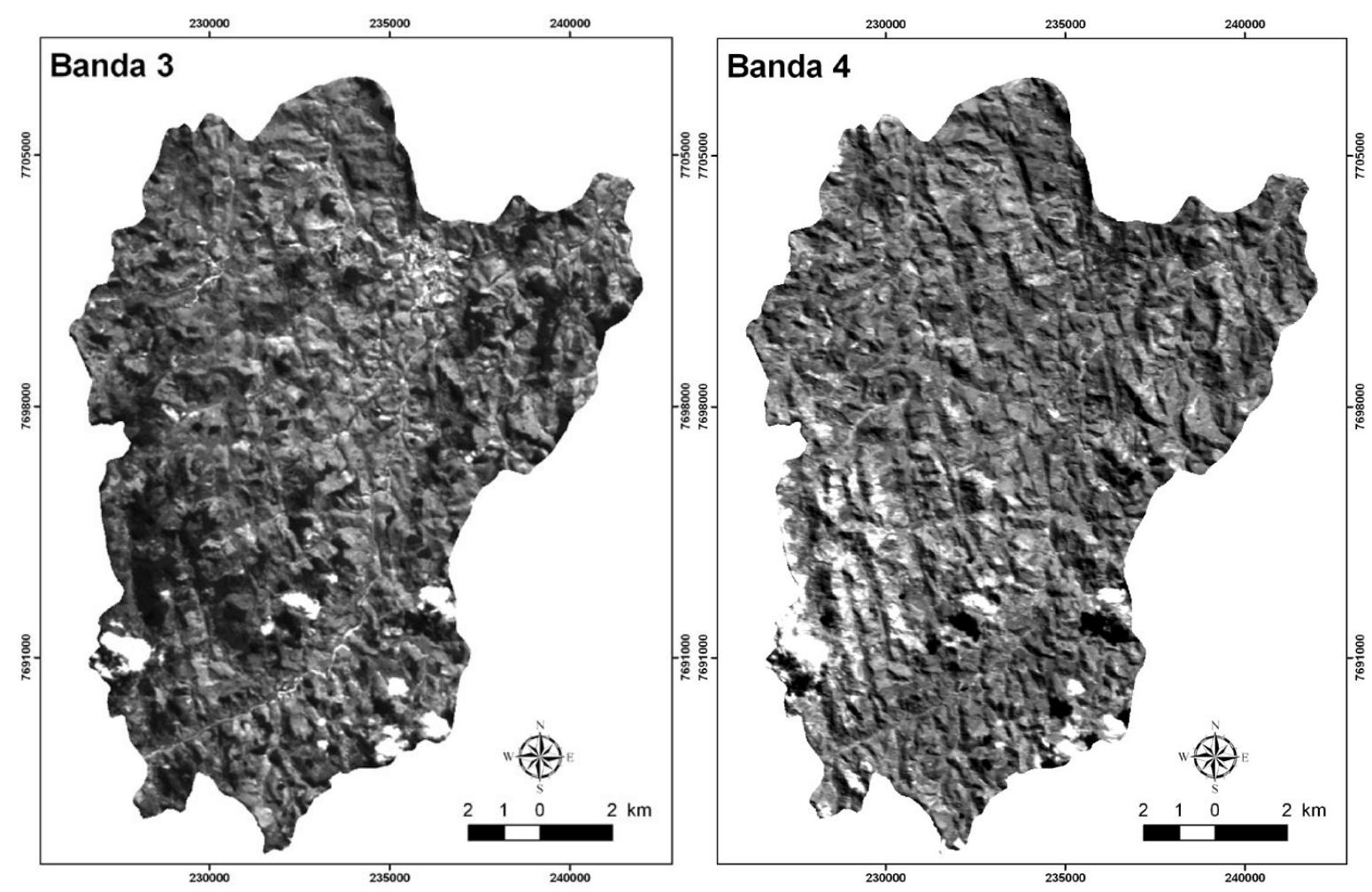

Figura 2 - Bandas 3 e 4 ajustadas.

O NDVI-04-08 obtido pela subtração das imagens NDVI-08 e NDVI-04, de acordo com a Equação 5, é apresentado na Figura 3. As tonalidades mais claras correspondem às áreas com cobertura vegetal; já os tons mais escuros indicam áreas de mudança ou desmatamento; e as tonalidades medianas correspondem às áreas que não sofreram alteração no período de estudo.

A mudança da cobertura vegetal ocorrida na bacia hidrográfica do Rio Alegre é ilustrada na Figura 4. As classes temáticas, desmatamento, não mudança e regeneração apresentaram 
áreas de 21,588 km² (10,26\%); $167,307 \mathrm{~km}^{2}(79,49 \%)$ e 21,582 km² (10,25\%), respectivamente. A classe de não mudança é predominante na região, demonstrando uma contínua redução dos desmatamentos, sendo explicado principalmente pelo fato da área já ter sido tomada há muitos anos pelo processo de urbanização e expansão das fronteiras agrícolas.

O desmatamento na bacia do rio Alegre está situado em maior parte na região Sudoeste, essa classe temática se consolida devido à presença intensiva da agricultura e pecuária. Por meio da análise do mapa temático (Figura 4), torna-se mais evidente a dinâmica de uso e ocupação do solo da bacia hidrográfica do Rio Alegre. As áreas das classes de regeneração e desmatamento possuem valores muito próximos, induzindo a uma condição de sustentabilidade na bacia, no entanto, o ideal seria comparar esses resultados com a realidade de campo.

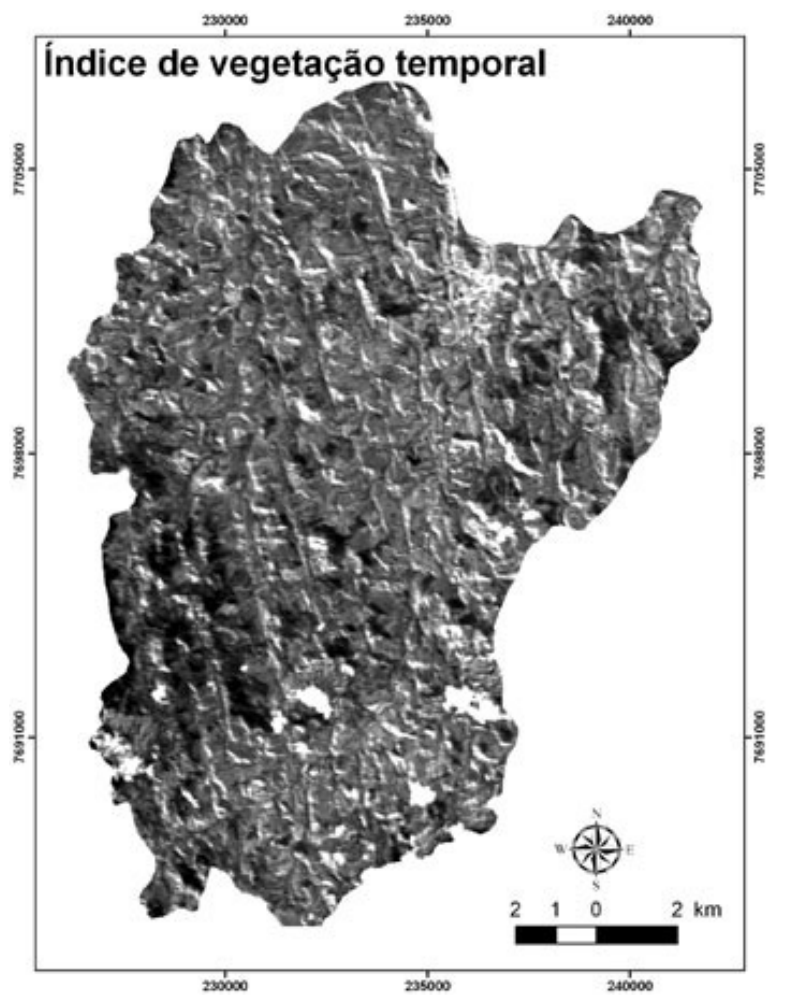

Figura 3- Evolução temporal do NDVI (período de 08/2004 e 07/2008). 


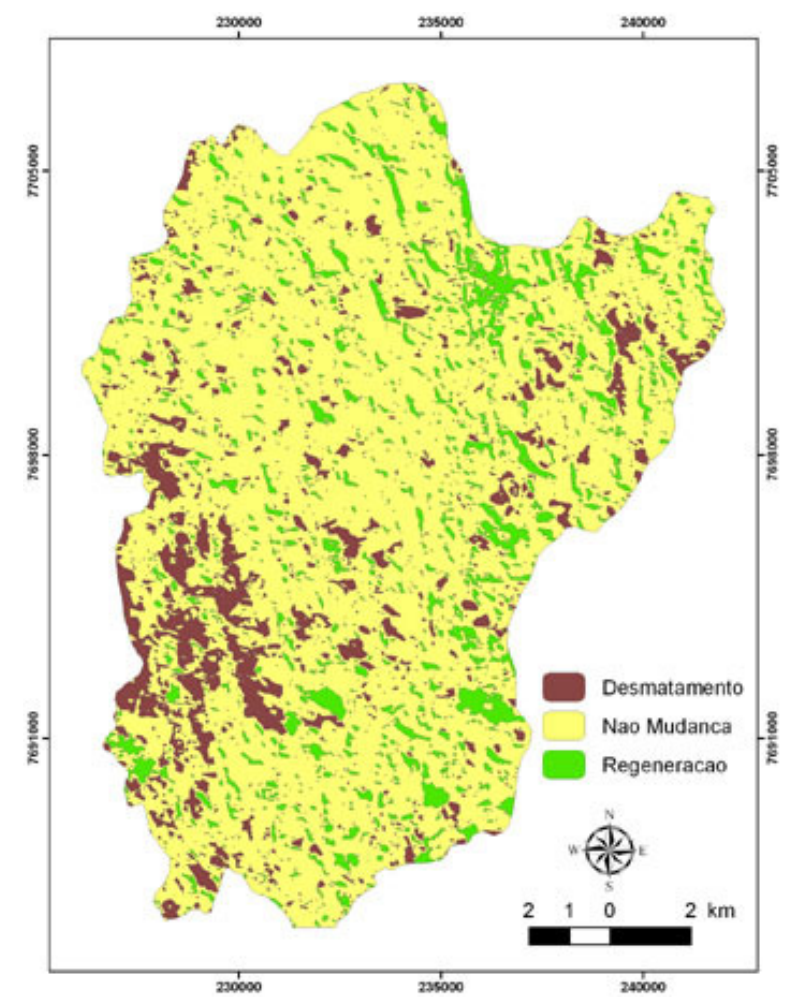

Figura 4- Mudança da cobertura da terra no período de 08/2004 a 07/2008.

\section{Conclusão}

A cobertura vegetal da bacia hidrográfica do rio Alegre se apresentou conservada ao longo dos quatro anos. Tal fato é ressaltado quando se observa a predominância da classe não mudança, acompanhada pela similaridade entre as áreas das classes de regeneração e desmatamento.

Como extensão deste trabalho, sugere-se que sejam realizadas análises da cobertura vegetal em períodos distintos e também futuros, a fim de verificar se áreas desmatadas e em regeneração continuarão apresentando as mesmas condições verificadas neste trabalho, e conseqüentemente sugerindo a sustentabilidade da bacia.

\section{Referências Bibliográficas}

Assad, E.D.; Castro, L.H.R.; Almeida, S.A.O. Análise exploratória da variação espaço temporal do índice de vegetação NOAA/AVHRR e pluviometria: caso dos cerrados brasileiros. In: Simpósio Brasileiro de Sensoriamento Remoto (SBSR), 8., 1996, Salvador. Anais... São José dos Campo: INPE, 1996. Artigos, p. 491493. CD-ROM, On-line. 85-17-00014-5. Disponível em: <http://marte.dpi.inpe.br/col/sid.inpe.br/deise/1999/02.04.14.42/doc/T30.pdf>. Acesso em: 10 nov. 2008.

Almeida, E.S.; Batista, G.T. Índice de vegetação versus precipitação na Amazônia. In: Simpósio Brasileiro de Sensoriamento Remoto (SBSR), 8., 1998, Santos. Anais... São José dos Campo: INPE, 1998. Artigos, p. 13971407. CD-ROM, On-line. $\quad 85-17-00015-3 . \quad$ Disponível em: <http://marte.dpi.inpe.br/col/dpi.inpe.br/lise/2004/07.20.14.26/doc/2_72o.pdf>. Acesso em: 10 nov. 2008.

Florenzano, T. G. Imagens de satélites para estudos ambientais. São Paulo: Oficina de Textos, 2002.97 p.

Justice, C.O.; Dugdale, G.; Townshend, J.R.G.; Narracott, A.S.; Kumar, M. Synergism between NOAA-AVHRR and Meteosat data for studying vegetation development in semi-arid West África. International Journal of Remote Sensing, v.12, n.6, p 1349-1368, 1991. 


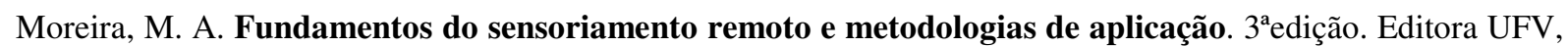
2005. 320p.

Nicholson, S.E.; Farrar, T.J. The influence of soil type on the relationships between NDVI, rainfall and soil moisture in semi-arid Botswna. Remote Sensing of Environment, v.50, p. 107 - 120, 1994.

Ponzoni, F. J. Comportamento espectral da vegetação. In: P. R. Meneses; J. S. M. Netto. Sensoriamento remoto: reflectância dos alvos naturais. Brasília: Editora Universidade de Brasília/Embrapa Cerrados, 2001. p. 157-199.

Potter, C.S.; Brooks, V. Global analysis of empirical relations between annual climate and seasonality of NDVI. International Journal of Remote Sensing, v.19, n.15, p. 2921-2948, 1998. 